\title{
COMMENT
}

\section{Understanding the impact of immune-mediated selection on lung cancer evolution}

\author{
Rachel Rosenthal (iD ${ }^{1,2}$, Charles Swanton ${ }^{1,2,3}$ and Nicholas McGranahan ${ }^{2,4}$
}

Understanding how a tumour evolves and avoids immune recognition is paramount to improving cancer immunotherapy and patient outcome. Here we examine our recent integration of multi-region genomic, transcriptomic, epigenomic, pathology, and clinical data, highlight the need for a systematic examination of immune escape mechanisms, and discuss implications for immunotherapy approaches.

British Journal of Cancer (2021) 124:1615-1617; https://doi.org/10.1038/s41416-020-01232-6

\section{MAIN}

Cancer is the result of an evolutionary process that relies on genetic diversity and is under selective pressures, including those from the immune microenvironment. The immune system may recognise antigens presented on the tumour cell, thus exerting an evolutionary pressure on the tumour and shaping the way it evolves. This can lead to the selection of subclonal populations of tumour cells that lack immunogenic antigens or are capable of withstanding an immune response. ${ }^{1,2}$

By analysing genomic, transcriptomic, epigenomic, pathology, and clinical data, it is possible to decipher a tumour's evolutionary history and the corresponding shifts in the immune contexture. This will allow a better understanding of the complex interplay between an evolving cancer and a dynamic immune microenvironment. In our recently published work, we performed an integrated analysis of 258 regions from 88 early-stage, untreated NSCLC from the "Tracking Non-Small-Cell Lung Cancer Evolution through Therapy" (TRACERx) clinical study. ${ }^{3,4}$ Using this cohort and its associated multi-region genomic, transcriptomic, epigenomic, pathology, and clinical data, we determined how levels of immune infiltration varied between and within tumours and subsequently characterised mechanisms acquired by the tumour that may have aided immune evasion and their associations with clinical outcome. $^{4}$

By clustering tumour regions based on their immune infiltration, we could classify tumours as being uniformly "immune cold", uniformly "immune hot", or heterogeneously infiltrated (if the same tumour had both "immune cold" and "immune hot" regions). Strikingly, we found that nearly a third of patients had tumours with disparate levels of immune infiltration between regions, highlighting a potentially confounding factor for the use of transcriptomic biomarkers in predicting response to immune-checkpoint blockade. This suggests a single tumour sample is often not representative of either the genomic landscape or the immune microenvironment of the tumour. ${ }^{5}$
Through previous work by our group and others, many immune-evasion mechanisms have been identified including: HLA loss or downregulation of expression, ${ }^{6-8}$ B2M loss and other antigen presentation defects, ${ }^{9}$ and the elimination of neoantigens through copy-number loss. ${ }^{10}$ We found that the majority of tumours harboured at least one mechanism of immune evasion, highlighting the strong selection pressure which the immune microenvironment imposes on a growing tumour. We also identified a novel epigenetic mechanism of immune evasion through neoantigen expression downregulation via promoter hypermethylation of genes that contain neoantigenic mutations.

Interestingly, we found that the mechanisms of neoantigen presentation dysfunction observed in a tumour differed according to its immune infiltration landscape. "Immune cold" tumours were enriched for copy-number loss of previously clonal neoantigens and showed a decrease in immunoediting during tumour evolution; "Immune hot" tumour regions exhibited either HLA loss of heterozygosity ( $\mathrm{LOH}$ ) or depletion of expressed neoantigens. This suggests an ongoing process of immune editing in highly infiltrated tumours and historical, or no longer active, immune editing in sparsely infiltrated tumours.

Finally, we developed the "immune-evasion capacity" metric to combine our assessment of the immune infiltration landscape with the identified immune-escape mechanisms in each tumour. Tumours with a low immune-evasion capacity were those that had high immune infiltration or no identified mechanism of immune escape. After determining this score for every patient's tumour, we observed that it could forecast disease-free survival-patients whose tumours had a low immune-evasion capacity exhibited significantly longer disease-free survival.

Taken together, our results highlight the dynamic nature of cancer evolution and the ongoing interplay between the cancer cell and the immune microenvironment. While our results provide only a single snapshot of the genomic, transcriptomic, and epigenetic landscape of the cancer cell, they suggest that the

\footnotetext{
${ }^{1}$ Cancer Evolution and Genome Instability Laboratory, The Francis Crick Institute, London, UK; ${ }^{2}$ Cancer Research UK Lung Cancer Centre of Excellence, University College London Cancer Institute, London, UK; ${ }^{3}$ Department of Medical Oncology, University College London Hospitals, London, UK and ${ }^{4}$ Cancer Genome Evolution Research Group, University College London Cancer Institute, University College London, London, UK

Correspondence: Rachel Rosenthal (rachel.rosenthal@crick.ac.uk)
}

Received: 15 September 2020 Revised: 20 November 2020 Accepted: 8 December 2020

Published online: 24 February 2021 
observed phenotype represents a product of continuous immune sculpting. These results may have important implications for existing immunotherapy and neoantigen vaccine approaches.

The observation of historic loss of neoantigens through copynumber events (analogous to a fossil record) in immune-cold regions raises the tantalising possibility that these tumour regions once were replete with immune cells. Conceivably, such immunecold regions could therefore be induced to become immune hot again, possibly through targeting neoantigens still present in the region or through modulating signalling molecules responsible for recruiting immunosuppressive cell types. ${ }^{11,12}$

Likewise, the observation that neoantigens may be silenced through promoter methylation suggests that these neoantigens could be re-activated through, for example, demethylating agents. Such an approach may also result in increased expression of major histocompatibility complex. Ongoing clinical trials are currently evaluating the effect of combining epigenetic modifiers with immune therapies. ${ }^{13}$ The transient nature of transcriptional repression and epigenetic silencing could also help explain why some tumours respond a second time following disease progression to checkpoint inhibitor blockade ${ }^{14}$ and highlight the importance of longitudinal and dynamic profiling, as currently only a single point in time of a tumour's immune/epigenomic landscape is considered.

A deeper understanding of the immune microenvironment and how it can change over time, both in the context of treatment-naive tumours and those treated with immunotherapy, is needed. These results also have implications for the design of neoantigen vaccines. In addition to the complexities already surrounding target neoantigen selection, such as mutation class, clonality, binding affinity, diversity of HLA presentation, antigen processing, and similarity to self, it will also be imperative to carefully evaluate the expression of putative neoantigen targets and, moreover, consider the likelihood of copy number or expression loss being selected as the tumour evolves. Further, they highlight the importance of integrating genomic and transcriptomic data to elucidate prime immune targets.

Finally, our study underscores the need to systematically examine different mechanisms of immune escape. Other mechanisms of expression downregulation, beyond promoter hypermethylation, certainly exist, which may be identified in larger cohorts, such as the expanded TRACERx cohort. Additional projects like RUBICON (a rule book and immune atlas for combination therapy) that aim to map out the immunological landscape and infiltrating cell types of TRACERx tumours in exhaustive detail will be instrumental in understanding precisely what makes a tumour region hot or cold and the complexity of the tumour/immune microenvironment.

\section{AUTHOR CONTRIBUTIONS}

R.R., N.M., and C.S. jointly conceived and wrote the manuscript.

\section{ADDITIONAL INFORMATION}

Ethics approval and consent to participate All results and patient data referenced in this study have been previously published.

\section{Consent to publish Not applicable.}

Data availability All data were published in https://www.nature.com/articles/s41586019-1032-7 under the EGA accession codes: EGAS00001003458 (RNA-seq) and EGAS00001003484 (RRBS).

Competing interests C.S. acknowledges grant support from Pfizer, AstraZeneca, Bristol Myers Squibb, Roche-Ventana, Boehringer-Ingelheim, Archer Dx Inc. (collaboration in minimal residual disease sequencing technologies) and Ono Pharmaceutical; is an
AstraZeneca Advisory Board Member and Chief Investigator for the MeRmaiD1 clinical trial; has consulted for Pfizer, Novartis, GlaxoSmithKline, MSD, Bristol Myers Squibb, Celgene, AstraZeneca, Illumina, Genentech, Roche-Ventana, GRAIL, Medicxi and the Sarah Cannon Research Institute; has stock options in Apogen Biotechnologies, Epic Bioscience and GRAIL; and has stock options and is co-founder of Achilles Therapeutics. C.S. holds European patents relating to assay technology to detect tumour recurrence (PCT/GB2017/053289); to targeting neoantigens (PCT/EP2016/059401), identifying patent response to immune checkpoint blockade (PCT/EP2016/071471), determining HLA LOH (PCT/GB2018/052004), predicting survival rates of patients with cancer (PCT/ GB2020/050221), identifying patients who respond to cancer treatment (PCT/GB2018/ 051912), a US patent relating to detecting tumour mutations (PCT/US2017/28013) and both a European and US patent related to identifying insertion/deletion mutation targets (PCT/GB2018/051892). N.M. and R.R. have stock options in and has consulted for Achilles Therapeutics and holds a European patent in determining HLA LOH (PCT/ GB2018/052004).

Funding information C.S. is a Royal Society Napier Research Professor. His work was supported by the Francis Crick Institute, which receives its core funding from Cancer Research UK (FC001169), the UK Medical Research Council (FC001169) and the Wellcome Trust (FC001169). C.S. is funded by Cancer Research UK (TRACERx, PEACE and CRUK Cancer Immunotherapy Catalyst Network), Cancer Research UK Lung Cancer Centre of Excellence, the Rosetrees Trust, Butterfield and Stoneygate Trusts, NovoNordisk Foundation (ID16584), Royal Society Research Professorships Enhancement Award (RP/EA/180007), the NIHR BRC at University College London Hospitals, the CRUK-UCL Centre, Experimental Cancer Medicine Centre and the Breast Cancer Research Foundation (BCRF). This research is supported by a Stand Up To Cancer-LUNGevity-American Lung Association Lung Cancer Interception Dream Team Translational Research Grant (SU2C-AACR-DT23-17). Stand Up To Cancer is a program of the Entertainment Industry Foundation. Research grants are administered by the American Association for Cancer Research, the Scientific Partner of SU2C. C.S. also receives funding from the European Research Council (ERC) under the European Union's Seventh Framework Programme (FP7/20072013) Consolidator Grant (FP7-THESEUS-617844), European Commission ITN (FP7PloidyNet 607722), an ERC Advanced Grant (PROTEUS) from the European Research Council under the European Union's Horizon 2020 research and innovation programme (835297) and Chromavision from the European Union's Horizon 2020 research and innovation programme (665233). N.M. is a Sir Henry Dale Fellow, jointly funded by the Wellcome Trust and the Royal Society (Grant Number 211179/Z/18/Z), and also receives funding from Cancer Research UK, Rosetrees and the NIHR BRC at University College London Hospitals and the CRUK University College London Experimental Cancer Medicine Centre.

Publisher's note Springer Nature remains neutral with regard to jurisdictional claims in published maps and institutional affiliations.

\section{REFERENCES}

1. Matsushita, H., Vesely, M. D., Koboldt, D. C., Rickert, C. G., Uppaluri, R., Magrini, V. J. et al. Cancer exome analysis reveals a T-cell-dependent mechanism of cancer immunoediting. Nature 482, 400-404 (2012).

2. Schreiber, R., Old, L. J. \& Smyth, M. J. Cancer immunoediting: integrating immunity's roles in cancer suppression and promotion. Science 331, 1565-1570 (2011).

3. Jamal-Hanjani, M., Wilson, G. A., McGranahan, N., Birkbak, N., Watkins, T. B. K., Veeriah, S. et al. Tracking the evolution of non-small-cell lung cancer. N. Engl. J. Med. 376, 2109-2121 (2017).

4. Rosenthal, R., Larose Cadieux, E., Salgado, R., Al Bakir, M., Moore, D. A., Hiley, C. T. et al. Neoantigen-directed immune escape in lung cancer evolution. Nature 567, 479-485 (2019).

5. Kather, J. N. \& Halama, N. Harnessing the innate immune system. Br. J. Cancer 120, 871-882 (2019)

6. Tran, E., Robbins, P. F., Lu, Y.-C., Prickett, T. D., Gartner, J. J., Jia, L. et al. T-cell transfer therapy targeting mutant KRAS in cancer. N. Engl. J. Med. 375, 2255-2262 (2016).

7. McGranahan, N., Rosenthal, R., Hiley, C. T., Rowan, A. J., Watkins, T. B. K., Wilson, G. A. et al. Allele-specific HLA loss and immune escape in lung cancer evolution. Cell 171, 1259-1271 (2017).

8. Garrido, F., Perea, F., Bernal, M., Sanchez-Palencia, A., Aptsiauri, N. \& Ruiz-Cabello, $F$. The escape of cancer from T cell-mediated immune surveillance: HLA class I loss and tumor tissue architecture. Vaccines 5, 7 (2017).

9. Drake, C. G., Jaffee, E. \& Pardoll, D. M. Mechanisms of immune evasion by tumors. Adv. Immunol. 90, 51-81 (2006). 
Understanding the impact of immune-mediated selection on lung cancer... $\mathrm{R}$ Rosenthal et al.

10. Anagnostou, V., Smith, K. N., Forde, P. M., Niknafs, N., Bhattacharya, R., White, J. et al. Evolution of neoantigen landscape during immune checkpoint blockade in non-small cell lung cancer. Cancer Discov. 7, 264-276 (2017).

11. Bonaventura, P., Shekarian, T., Alcazer, V., Valladeau-Guilemond, J., ValsesiaWittmann, S., Amigorena, S. et al. Cold tumors: a therapeutic challenge for immunotherapy. Front. Immunol. 10, 168 (2019).

12. Li, J., Byrne, K. T., Yan, F., Yamazoe, T., Chen, Z., Baslan, T. et al. Tumor cell-intrinsic factors underlie heterogeneity of immune cell infiltration and response to immunotherapy. Immunity 49, 178.e7-193.e7 (2018).
13. Aspeslagh, S., Morel, D., Soria, J.-C. \& Postel-Vinay, S. Epigenetic modifiers as new immunomodulatory therapies in solid tumours. Ann. Oncol. 29, 812-824 (2018).

14. Borcoman, E., Nandikolla, A., Long, G., Goel, S. \& Le Tourneau, C. Patterns of response and progression to immunotherapy. Am. Soc. Clin. Oncol. Educ. Book 38, 169-178 (2018). 